\title{
Salt supplementation blunts the blood pressure response to telmisartan with or without hydrochlorothiazide in hypertensive patients with type 2 diabetes
}

\author{
E. I. Ekinci • G. Thomas • R. J. MacIsaac • C. Johnson • \\ C. Houlihan • S. Panagiotopoulos • E. Premaratne • \\ H. Hao • S. Finch • C. O'Callaghan • G. Jerums
}

Received: 5 December 2009 / Accepted: 27 January 2010/Published online: 7 April 2010

(C) Springer-Verlag 2010

\begin{abstract}
Aims/hypothesis We assessed the effects of sodium chloride $(\mathrm{NaCl})$ supplementation on the blood pressure response to treatment with telmisartan with or without hydrochlorothiazide in hypertensive patients with type 2 diabetes and habitually high (HDS, sodium excretion $>200 \mathrm{mmol} / 24 \mathrm{~h}$ on two out of three consecutive occasions) or low (LDS, sodium excretion $<100 \mathrm{mmol} / 24 \mathrm{~h}$ on two out of three consecutive occasions) salt intake.

Methods Patients received 4 weeks of telmisartan followed by 4 weeks of telmisartan plus hydrochlorothiazide. In a double-blind randomised fashion, patients received sodium chloride $(\mathrm{NaCl}, 100 \mathrm{mmol} / 24 \mathrm{~h})$ or placebo capsules in
\end{abstract}

Electronic supplementary material The online version of this article (doi:10.1007/s00125-010-1711-2) contains supplementary material, which is available to authorised users.

E. I. Ekinci $(\bowtie) \cdot$ G. Thomas $\cdot$ R. J. MacIsaac $\cdot$ C. Johnson •

C. Houlihan $\cdot \mathrm{S}$. Panagiotopoulos $\cdot$ E. Premaratne $\cdot$ G. Jerums

Department of Endocrinology,

Austin Health and the University of Melbourne,

Level 2 Centaur Building, Repatriation Campus, Austin Health,

Heidelberg West,

Melbourne, VIC 3081, Australia

e-mail: eekinci2002@yahoo.com.au

H. Hao

Vascular Laboratory, Austin Health,

Melbourne, VIC, Australia

S. Finch

Statistical Consulting Service, The University of Melbourne,

Melbourne, VIC, Australia

C. O’Callaghan

Department of Clinical Pharmacology, Austin Health,

Melbourne, VIC, Australia addition to their habitual salt intake during the last 2 weeks of telmisartan and telmisartan plus hydrochlorothiazide therapy. The protocol was repeated with $\mathrm{NaCl}$ and placebo capsules administered in reverse order to allow each participant to act as his or her own control. At $0,4,8,14$, 18 and 22 weeks, $24 \mathrm{~h}$ ambulatory blood pressure (ABP) and $24 \mathrm{~h}$ urine collections were performed.

Results No statistically significant differences were seen in the ABP response in the LDS vs HDS groups to any of the interventions $(p=0.58) . \mathrm{NaCl}$ supplementation reduced the effect of telmisartan with or without hydrochlorothiazide on systolic BP by approximately $50 \%(-5.8 \mathrm{mmHg}$ during $\mathrm{NaCl}$ supplementation vs $-11.3 \mathrm{mmHg}$ during placebo, mean difference $5.6 \mathrm{mmHg}$ [95\% CI $1.7-9.4 \mathrm{mmHg}$ ], $p=$ 0.005), irrespective of habitual salt intake. By contrast, addition of hydrochlorothiazide increased the antihypertensive effect of telmisartan on systolic BP by approximately $35 \%(p=0.048)$ in both groups of patients.

Conclusions/interpretation $\mathrm{NaCl}$ supplementation blunts the effectiveness of telmisartan with or without hydrochlorothiazide in hypertensive patients with type 2 diabetes, independently of habitual low or high salt intake.

Trial registration Australian Clinical Trials Registry ACTRN012606000128594

Funding This work was supported by a Pfizer CardiovascularLipid Research Grant and an unconditional educational grant from Boehringer Ingelheim. During the period of this work, E. I. Ekinci was supported by grants from the Austin Hospital Medical Research Foundation and the National Health and Medical Research Council.

Keywords Angiotensin receptor blockade - Hypertension Plasma renin activity - Salt . Sodium . Thiazide diuretic therapy Type 2 diabetes 


\begin{tabular}{|c|c|}
\hline \multicolumn{2}{|c|}{ Abbreviations } \\
\hline $\mathrm{ABP}$ & $24 \mathrm{~h}$ Ambulatory blood pressure \\
\hline HCT & Hydrochlorothiazide \\
\hline $\mathrm{NaCl}$ & $\begin{array}{l}\text { Sodium chloride supplementation, } 100 \mathrm{mmol} \mathrm{NaCl} \\
24 \mathrm{~h}\end{array}$ \\
\hline HDS & $\begin{array}{l}\text { Habitual high dietary salt intake, sodium excretion } \\
>200 \mathrm{mmol} / 24 \mathrm{~h} \text { on two out of three consecutive } \\
\text { occasions }\end{array}$ \\
\hline LDS & $\begin{array}{l}\text { Habitual low dietary salt intake, sodium excretion } \\
<100 \mathrm{mmol} / 24 \mathrm{~h} \text { on two out of three consecutive } \\
\text { occasions }\end{array}$ \\
\hline MAP & Mean arterial pressure \\
\hline RAS & Renin-angiotensin system \\
\hline AER & Albumin excretion rate \\
\hline
\end{tabular}

\section{Introduction}

The management of elevated blood pressure is an important public health challenge, especially in patients with type 2 diabetes [1, 2]. Even in the setting of antihypertensive clinical trials, the blood pressure levels achieved commonly exceed [3] the Joint National Committee-VII target of $\leq 130 / 80 \mathrm{mmHg}$ [4]. This suggests that there is scope to improve the management of elevated blood pressure in people with diabetes.

Evidence from epidemiological, migration, intervention and experimental hypertension studies indicates that salt intake plays an important role in regulating blood pressure $[5,6]$. Excess sodium and a deficit of potassium have been implicated in the generation of increased arterial and arteriolar smooth muscle tone in primary hypertension [7]. In comparison to people without diabetes, patients with diabetes have an increase in exchangeable sodium, which correlates with systolic blood pressure [8,9]. Dietary salt intake may therefore have a more important role in the pathogenesis of hypertension in diabetes than in the general population.

We hypothesised that $\mathrm{NaCl}$ supplementation, due to its effects on the activity of the systemic renin-angiotensin system (RAS) might influence the antihypertensive actions of telmisartan with and without hydrochlorothiazide (HCT) differently in groups with habitual high dietary salt (HDS) and habitual low dietary salt (LDS) intake. We therefore performed a double blind, prospective, randomised crossover study of the effects of $\mathrm{NaCl}$ supplementation on the response to telmisartan with or without HCT, in subjects with type 2 diabetes, hypertension, raised albumin excretion rate (AER) and habitual high or low salt intake. In this study we found that the AER response was blunted by $\mathrm{NaCl}$ supplementation only in subjects with habitual low salt intake [10]. Here we describe in detail the blood pressure response to telmisartan with or without HCT in the same group of patients and show that the effect of $\mathrm{NaCl}$ supplementation on blood pressure is independent of habitual salt intake.

\section{Methods}

The methods for this study have been described in detail elsewhere [10]. In brief, 32 patients with type 2 diabetes, hypertension and AER in the range of 10 to $200 \mu \mathrm{g} / \mathrm{min}$ were recruited from diabetes clinics at Austin Health, a tertiary referral centre where patients routinely perform a $24 \mathrm{~h}$ urine collection for the measurement of AER and sodium excretion. Eligibility criteria for participation in the study were: a history of type 2 diabetes, hypertension (blood pressure $>140 / 90 \mathrm{mmHg}$ or taking antihypertensive medication), AER between 10 and $200 \mu \mathrm{g} / \mathrm{min}$ (median of three consecutive measurements collected over a 12 month period), and either habitually high or low sodium intake (HDS: sodium excretion $>200 \mathrm{mmol} / 24 \mathrm{~h}$ on two out of three consecutive occasions; LDS: $<100 \mathrm{mmol} / 24 \mathrm{~h}$ on two out of three consecutive occasions). Patients in HDS and LDS groups were matched for BMI because of the documented association between BMI and urinary sodium excretion [11].

Exclusion criteria were serum potassium $>5.0 \mathrm{mmol} / \mathrm{l}$, serum creatinine $>200 \mu \mathrm{mol} / \mathrm{l}, \mathrm{AER}>200 \mu \mathrm{g} / \mathrm{min}, \mathrm{HbA}_{1 \mathrm{c}}>$ $10.0 \%$ and major systemic illness. All patients gave informed consent before commencement of the study, which was approved by the Human Research Ethics Committee at Austin Health. The procedures followed were in accordance with institutional guidelines.

Study protocol The study was undertaken in six phases (Fig. 1). Phase 1 comprised a 6 week washout period, during which usual dietary sodium intake was continued and patients were randomised by computer into two groups (A or B) by the hospital pharmacy department. Drugs interfering with the RAS and diuretics were ceased at -6 weeks and replaced with verapamil, prazosin, methyldopa and/or hydralazine as necessary to achieve a blood pressure $<160 / 95 \mathrm{mmHg}$. Dose adjustments of these drugs were then made between weeks -6 and -3 . At week 0 , all patients commenced treatment with telmisartan $40 \mathrm{mg}$ for 4 weeks (Phase 2). This was followed by telmisartan (40 mg) plus HCT $(12.5 \mathrm{mg}$ ) for an additional 4 weeks (Phase 3). Patients in group A received placebo capsules in addition to their usual salt intake during the last 2 weeks of telmisartan monotherapy and during the last 2 weeks of combined telmisartan and hydrochlorothiazide therapy, whereas patients in group $\mathrm{B}$ received $\mathrm{NaCl}$ capsules under otherwise identical conditions. Phase 4 consisted of a 


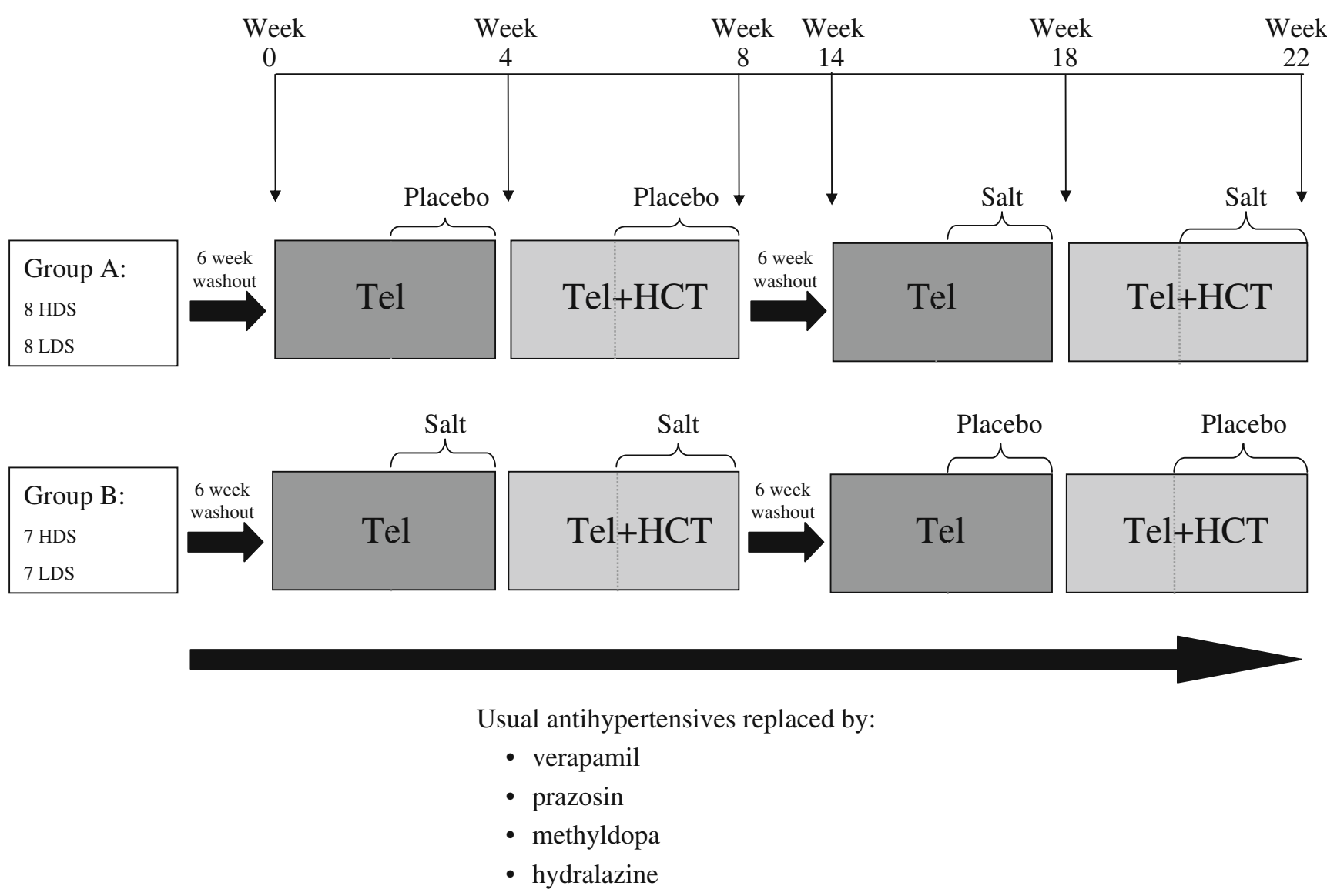

Fig. 1 Study protocol. Patients with HDS and LDS were randomised to Group A or Group B. Patients took telmisartan (Tel) for 4 weeks followed by Tel+HCT for 4 weeks. In the last 2 weeks, placebo or

6 week washout period for telmisartan, $\mathrm{HCT}$, and $\mathrm{NaCl}$ or placebo capsules. Patients remained on the same doses of background non-RAS-interfering antihypertensive drugs throughout the study. Following the second washout period (phase 4), the protocol was repeated with $\mathrm{NaCl}$ and placebo capsules administered in reverse order (phases 5 and 6) (Fig. 1). Patients were instructed to take their antihypertensive medications after breakfast. Investigators and patients were blinded to the identity of participants receiving $\mathrm{NaCl}$ or placebo capsules. $\mathrm{NaCl}$ and placebo capsules were manufactured by a local registered compounding pharmacy (Thompson's Pharmacy, Eltham, VIC, Australia). Five capsules, each containing $20 \mathrm{mmol} \mathrm{NaCl}$ (total $100 \mathrm{mmol} \mathrm{NaCl}$ ) or placebo (lactose) were administered daily. Participants were instructed to take two capsules with breakfast, one with lunch and two with the evening meal.

Laboratory or diagnostic procedures were performed as follows: (1) 24-h ambulatory blood pressure (ABP) measurements at $0,4,8,14,18,22$ weeks; (2) 24 h urine collection to measure albumin, sodium, potassium, glucose and creatinine excretion rates at $0,4,8,14,18$ and 22 weeks; (3) fasting blood sample for the measurement
$\mathrm{NaCl}$ capsules were added. Following a second washout period, the protocol was repeated with $\mathrm{NaCl}$ and placebo capsules administered in reverse order

of plasma glucose, plasma renin activity, aldosterone (after patients had been seated for $5 \mathrm{~min}$ ), $\mathrm{HbA}_{1 \mathrm{c}}$, creatinine and electrolyte levels at $0,4,8,14,18,22$ weeks. For the $24 \mathrm{~h}$ urine collection, patients were given instructions on how to perform collections accurately, and urinary sodium excretion was corrected by adjusting for average creatinine excretion in the six urine samples for each patient.

All samples for biochemical analyses were collected in the morning after an overnight fast and before the administration of study medication. The study dietitian (C. Johnson) saw all patients initially and gave them advice, based on their previous diet, to continue with their habitual sodium intake.

Ambulatory blood pressure measurements The $24 \mathrm{~h}$ ABP was conducted with a portable recording system (Spacelabs 90207; Spacelabs Medical Products, Deerfield, WI, USA) based on an oscillometric method. The $24 \mathrm{~h}$ systolic, diastolic and mean arterial pressures (MAP), as well as awake and sleep times were recorded. Blood pressure was measured every $30 \mathrm{~min}$ from 07:00 hours to 23:00 hours and every hour from 23:00 hours to 07:00 hours. Mean arterial blood pressure was defined as diastolic BP $+1 / 3$ (pulse pressure). 
Statistical analysis In a previous study from our laboratory [12], the difference in mean ABP taken at two measurements 8 weeks apart in ten participants was $2 \pm 5.5 \mathrm{mmHg}$ (mean $\pm \mathrm{SD}$ ). To detect a difference in blood pressure response of one SD with a power of $90 \%$ and an alpha of $5 \%$, we estimated that paired data from 15 participants would be required for this crossover study design [13]. Therefore we planned to recruit 16 participants in both the HDS and LDS groups.

Statistical analysis used ANOVA models run on Genstat (10th edition; VSN International, Hemel Hempstead, UK). Factors relating to the crossover design were phases of the study (2-3 or 5-6) and randomisation sequence group (group A or group B). Three factors of interest were diet group and the two within-patient factors $\mathrm{NaCl}$ vs placebo and telmisartan vs telmisartan + HCT. Outcomes included attained blood pressure (systolic, mean, diastolic) and change in blood pressure (systolic, mean, diastolic) from baseline. A logarithmic transformation was applied to plasma renin activity to satisfy the requirements of the ANOVA models.

For each outcome, two models were considered. The first model included main effects of the crossover design factors in addition to the three factors of interest; the second model did not. We report results based on the second model, as we found no evidence of crossover effects. The main effects of habitual diet group, i.e. $\mathrm{NaCl}$ vs placebo supplementation and telmisartan vs telmisartan $+\mathrm{HCT}$, are described in this paper. None of the higher order interactions were statistically significant. We also considered a model that included the effects of hydralazine and verapamil; these effects were trivial and hence we report the more parsimonious analyses without these effects.

\section{Results}

Participants Of the 32 participants, 17 were recruited to the HDS group and 15 to the LDS group over a period of 9 months between May 2006 and January 2007 (Electronic supplementary material [ESM] Fig. 1). Three patients from the HDS group were withdrawn from the study. Two of the patients were unable to meet the attendance requirements of the study and one was unable to tolerate salt capsules. All other participants completed the study despite some minor gastrointestinal side effects from the $\mathrm{NaCl}$ capsules.

Baseline characteristics The baseline characteristics of the study population have been outlined previously [10] and are summarised in Table 1. There were no significant differences in the baseline clinical and biochemical variables between HDS and LDS participants, except that there were more men in the HDS group ( $p=0.006$ ). No difference in baseline MAP was observed in the HDS and LDS groups (HDS $101 \pm 2.2 \mathrm{mmHg}$, LDS $98 \pm 2.2 \mathrm{mmHg}, p=0.24$ ) and there was no evidence of a crossover effect from phase 1 to phase 4. As expected, 24 urinary sodium excretion was higher in the HDS compared with the LDS group (HDS, $271 \pm 24 \mathrm{mmol} / 24 \mathrm{~h}$, LDS $118 \pm 12 \mathrm{mmol} / 24 \mathrm{~h}, p<0.0001$ ). There was no difference in baseline AER between the HDS and the LDS groups (HDS $34 \times / \div 1.3,56 \times / \div$ $1.4 \mu \mathrm{g} / \mathrm{min}$ (geometric mean $\times / \div$ tolerance factor, $p=$ $0.3)$ ). There was also no difference in the number of patients requiring additional antihypertensives to achieve target blood pressure of $<140 / 90 \mathrm{mmHg}$ (HDS $n=11$, LDS $n=8 ; p=0.49$ ).

Urinary sodium excretion The $24 \mathrm{~h}$ urinary sodium excretion rates for the different phases of the study are shown in Fig. 2a for LDS and HDS patients. The mean difference in urinary sodium excretion during the entire study between these two groups was $136 \mathrm{mmol} / 24 \mathrm{~h}(95 \%$ CI 99-174) $(p<0.001)$ (ESM Table 1).

In the LDS group, mean urinary sodium excretion during placebo vs $\mathrm{NaCl}$ supplementation was $126 \mathrm{mmol} / 24 \mathrm{~h}$ (95\% CI 96-156), vs 176 mmol/24 h (95\% CI 146-206), respectively. In the HDS group, mean urinary sodium excretion during placebo vs $\mathrm{NaCl}$ supplementation was $256 \mathrm{mmol} / 24 \mathrm{~h}$ ( $95 \%$ CI $225-286)$ vs $318 \mathrm{mmol} / 24 \mathrm{~h}(95 \%$ CI 287-349), respectively. Overall, $\mathrm{NaCl}$ supplementation increased urinary sodium excretion by $56 \mathrm{mmol} / 24 \mathrm{~h}(95 \%$ CI 34-78). There was no significant increase in urinary sodium excretion after addition of HCT to telmisartan in the combined analysis of HDS and LDS groups, i.e. an increase of only $2.8 \mathrm{mmol} / 24 \mathrm{~h}(95 \%$ CI $19-24 \mathrm{mmol} / 24 \mathrm{~h})(p=0.8)$.

Table 1 Baseline characteristics of study participants

\begin{tabular}{|c|c|}
\hline Variables & Values \\
\hline$N$ & 29 \\
\hline Age (years) & $62.2 \pm 1.6$ \\
\hline BMI $\left(\mathrm{kg} / \mathrm{m}^{2}\right)$ & $32.5 \pm 1.1$ \\
\hline $\mathrm{HbA}_{1 \mathrm{c}}(\%)$ & $7.3 \pm 0.2$ \\
\hline Smoker:non-smoker $(n: n)$ & $5: 24$ \\
\hline Systolic BP (mmHg) & $139 \pm 3$ \\
\hline Diastolic BP (mmHg) & $78 \pm 2$ \\
\hline Serum creatinine $(\mu \mathrm{mol} / \mathrm{l})$ & $88.0 \pm 3.6$ \\
\hline Estimated GFR $\left(\mathrm{ml} \min ^{-1}\left[\begin{array}{ll}1.73 & \mathrm{~m}\end{array}\right]^{-2}\right)$ & $78.2 \pm 3.4$ \\
\hline Baseline AER $(\mu \mathrm{g} / \mathrm{min})^{\mathrm{a}}$ & $43.4 \times / \div 1.3$ \\
\hline Patients requiring additional antihypertensives $(n)^{\mathrm{b}}$ & 19 \\
\hline
\end{tabular}

Results are shown as mean \pm SEM except where indicated otherwise

${ }^{\text {a }}$ AER shown as geometric mean $\times / \div$ tolerance factor

${ }^{\mathrm{b}}$ To achieve target blood pressure $<140 / 90 \mathrm{mmHg}$ 


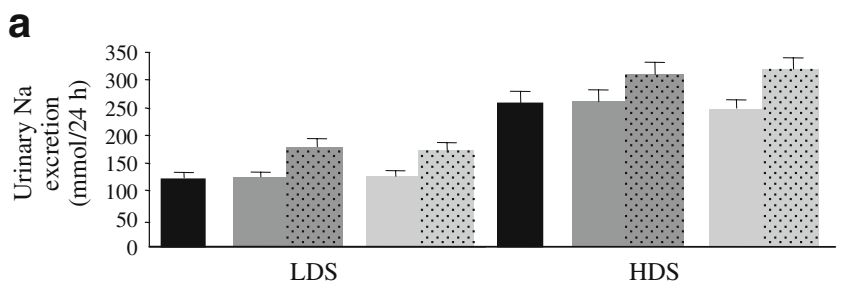

b

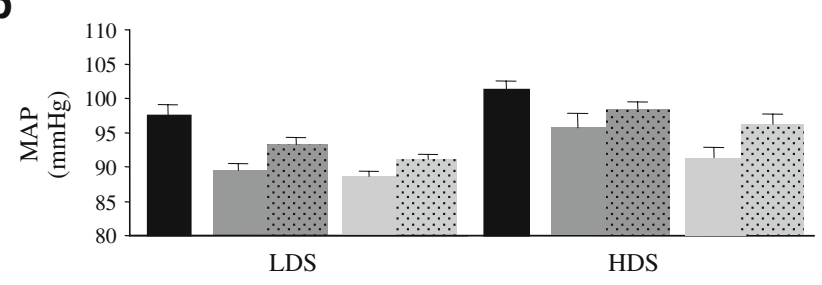

C

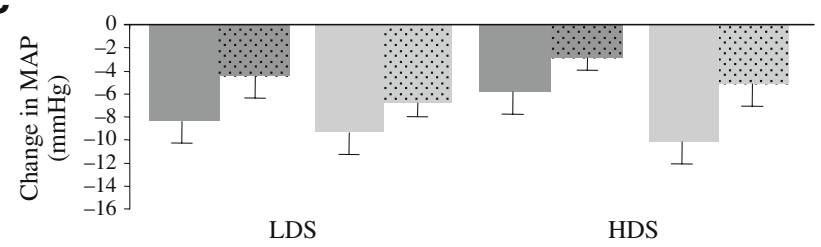

Fig. 2 Attained urinary sodium excretion (a), attained mean arterial pressure (MAP) (b) and change in MAP (c). Data were analysed by a three-way ANOVA. The three factors analysed were: HDS vs LDS; $\mathrm{NaCl}$ supplement vs placebo capsules; and telmisartan vs telmisartan+ HCT treatment. Black, baseline; dark grey, telmisartan; light grey, telmisartan $+\mathrm{HCT}$; dotted bars, $\mathrm{NaCl}$ supplementation (a) Urinary sodium excretion: HDS vs LDS, $p<0.001$; $\mathrm{NaCl}$ vs placebo, $p<0.001$; telmisartan vs telmisartan $+\mathrm{HCT}, p=0.8$. (b) Attained MAP: HDS vs LDS, $p=0.062 ; \mathrm{NaCl}$ vs placebo, $p<0.001$; telmisartan vs telmisartan + HCT, $p=0.019$. (c) Change in MAP: HDS vs LDS, $p=0.6$; $\mathrm{NaCl}$ vs placebo, $p=0.007$; telmisartan vs telmisartan + HCT, $p=0.054$

No evidence of a higher order interaction was found that might have suggested a differential effect of $\mathrm{NaCl}$ supplementation in the two habitual dietary sodium groups ( $p=$ 0.6) (ESM Table 1).

Urinary potassium excretion Urinary potassium excretion in the HDS group was greater than in the LDS group (mean difference in potassium excretion between two groups: $26.8 \mathrm{mmol} /$ day [95\% CI $9-45 \mathrm{mmol} / \mathrm{day}], p=0.005$ ). Urinary sodium:potassium ratio was higher in the HDS group (mean difference between HDS and LDS: 0.97 [95\% CI $0.32-1.61], p=0.005)$. Urinary potassium excretion correlated with urinary sodium excretion $(r=0.45$ [95\% CI $0.32-0.57], p<0.0001)$.

Effects on attained blood pressure Both $\mathrm{NaCl}$ supplementation and HCT influenced the attained blood pressure level, but in opposite directions in the LDS and HDS groups (Table 2, ESM Table 2). Although there was a trend to higher attained MAP in the HDS group $(p=0.06)$, habitual dietary salt intake did not significantly alter the effects of therapy on attained blood pressure or changes in blood pressure levels (ESM Tables 1 and 2).
Effects of $\mathrm{NaCl}$ supplementation on response to therapy The overall analysis of HDS and LDS patients showed that $\mathrm{NaCl}$ supplementation reduced the effect of telmisartan with or without HCT on the change in systolic BP from baseline by approximately $50 \%$, regardless of whether telmisartan or telmisartan plus HCT was administered ( $\mathrm{NaCl}-5.8 \mathrm{mmHg}$ vs placebo $-11.3 \mathrm{mmHg}$, mean difference 5.6 [95\% CI 1.7-9.4] mmHg, $p=0.005$ ) (Table 2, ESM Tables 2 and 3). Similarly, $\mathrm{NaCl}$ supplementation reduced the effect of telmisartan with or without HCT on changes in MAP $(\mathrm{NaCl}-4.9 \mathrm{mmHg}$ vs placebo $-8.4 \mathrm{mmHg}$, mean difference 3.5, [95\% CI 1.0-6.0] $\mathrm{mmHg}, p=0.007$ ) (ESM Table 3). However, $\mathrm{NaCl}$ supplementation had no significant effect on changes in diastolic blood pressure levels during therapy with telmisartan with or without HCT (mean difference $1.8 \mathrm{mmHg}$ [95\% CI 3.80.2 ], $p=0.08)$ (ESM Table 3).

The overall analysis of HDS and LDS patients also revealed that $\mathrm{NaCl}$ supplementation resulted in a higher attained MAP of $94.6 \mathrm{mmHg}$ (95\% CI 93.1-96.0), compared with $91.0 \mathrm{mmHg}(95 \%$ CI 89.6-92.5, $p<0.001)$ (ESM Table 2) for placebo treatment. As shown in Fig. 2a and b, the attained MAP levels generally reflected the urinary sodium excretion in the different phases of the study. The attained systolic BP was higher during $\mathrm{NaCl}$ supplementation $(132.6 \mathrm{mmHg}$ [95\% CI $130.5-134.8]$ vs placebo $128.1 \mathrm{mmHg}$ [95\% CI 125.9-130.2], $p=0.004$ ) (ESM Table 2). The attained diastolic $\mathrm{BP}$ was also higher during $\mathrm{NaCl}$ supplementation, i.e. $73.5 \mathrm{mmHg}(95 \%$ CI $72.4-74.6)$ vs placebo $71.2 \mathrm{mmHg}$ (95\% CI 70.0-72.3), $p=0.005$ (ESM Table 2).

Effects of adding HCT to telmisartan in high and low sodium intake groups In the overall analysis of HDS and LDS groups, addition of HCT to telmisartan increased the systolic BP response by approximately $35 \%$, i.e. $-6.6 \mathrm{mmHg}$ (telmisartan alone) vs $-10.5 \mathrm{mmHg}$ (telmisartan plus HCT), mean difference $3.9 \mathrm{mmHg}(95 \%$ CI $0.03-$ 7.7), $p=0.048$ (Table 2, Fig. 3b, ESM Table 3). This result was irrespective of $\mathrm{NaCl}$ supplementation.

Similarly, the overall analysis of HDS and LDS groups showed that the MAP attained was lower with telmisartan plus HCT (91.6 mmHg [95\% CI 90.1-93.0]) compared with telmisartan alone $(94.0 \mathrm{mmHg}$ [95\% CI 92.6-95.5], $p=0.02$ ) (ESM Table 2). The systolic BP attained was lower with telmisartan plus HCT $(128.4 \mathrm{mmHg}$ [95\% CI 126.3-130.6]) than with telmisartan alone $(132.3 \mathrm{mmHg}$ [95\% CI 130.1-134.40], $p=0.01$ ) (Table 2, ESM Table 2). However, the diastolic BP attained was not significantly affected by adding HCT to telmisartan therapy $(71.7 \mathrm{mmHg}$ [95\% CI 70.6-72.8] vs $72.9 \mathrm{mmHg}$ [95\% CI 71.8-74.1] with telmisartan alone, $p=0.13$ ) (Table 2, ESM Table 2).

In the study as a whole, the attenuation of the systolic BP response with $\mathrm{NaCl}$ supplementation $(5.6 \mathrm{mmHg}$ [95\% 
Table 2 Attained blood pressure and change in blood pressure $(\mathrm{mmHg})$ from baseline according to treatment group

$p=0.004$ and $p=0.005$ for $\mathrm{NaCl}$ supplement vs placebo in systolic and diastolic BP respectively; $p=0.01$ and $p=0.13$ for telmisartan vs telmisartan $+\mathrm{HCT}$ in systolic and diastolic BP, respectively; HDS vs LDS systolic $\mathrm{BP} p=0.06$ and diastolic BP $p=0.34$

Tel, telmisartan

\begin{tabular}{lcccc}
\hline BP per group & Tel+placebo & Tel+NaCl & Tel+HCT + placebo & Tel+HCT+NaCl \\
\hline Systolic BP & & & & \\
LDS, attained & 125.3 & 130.7 & 123.4 & 127.5 \\
LDS, change & -10.5 & -4.3 & -12.3 & -7.4 \\
HDS, attained & 136.1 & 137.7 & 128.1 & 135.1 \\
HDS, change & -7.3 & -4.4 & -15.3 & -7.1 \\
Diastolic BP & & & & \\
LDS, attained & 70.3 & 72.4 & 69.8 & -5.7 \\
LDS, change & -7.0 & -5.0 & -7.5 & 74.6 \\
HDS, attained & 73.8 & 75.5 & 70.9 & -4.6 \\
HDS, change & -4.4 & -3.7 & -7.3 & \\
\hline
\end{tabular}

CI $1.7-9.4 \mathrm{mmHg}], p=0.005)$ was greater than the antihypertensive effect of adding $\mathrm{HCT}$ to telmisartan (3.9 mmHg [95\% CI 0.03-7.7], $p=0.048$ ) (Fig. 3, ESM Table 3) in telmisartan-treated patients.

Plasma renin activity This was significantly higher throughout the study in the LDS group than in the HDS group ( $p=0.046)$ (ESM Tables 1 and 4). In both HDS and LDS groups, plasma renin activity was significantly suppressed with $\mathrm{NaCl}$ supplementation, compared with placebo $(p<0.001)$ (ESM Tables 1 and 4). Moreover, addition of HCT to telmisartan significantly increased plasma renin activity in both HDS and LDS groups $(p<0.001)$ (ESM Tables 1 and 4).
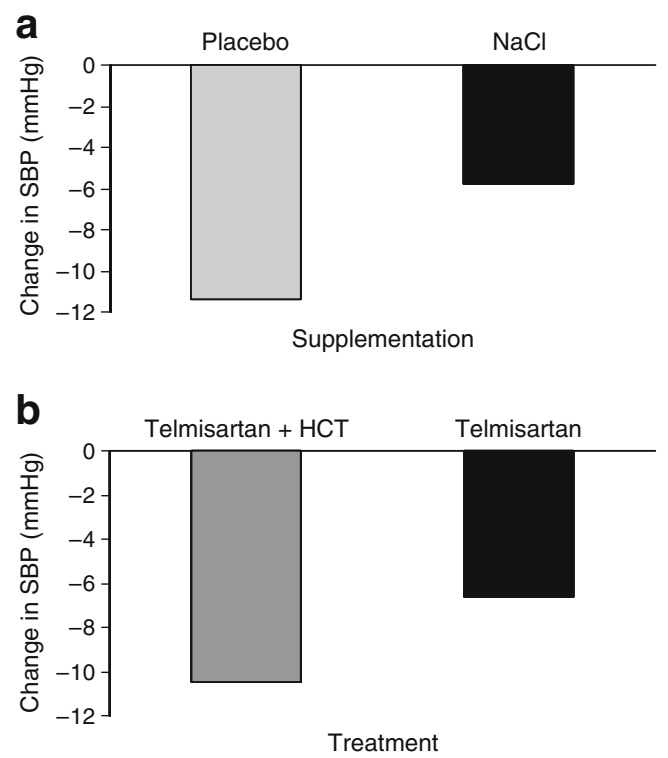

Fig. 3 The effects on systolic $\mathrm{BP}$ of $\mathrm{NaCl}$ supplementation (a), and addition of HCT to telmisartan therapy (b), in the combined analysis of HDS and LDS groups. (a) Light grey bar, placebo supplementation; black bar, $\mathrm{NaCl}$ supplementation. (b) Dark grey bar, telmisartan+ HCT; black bar, telmisartan $+\mathrm{HCT}$
Changes in albumin excretion rate The changes in AER in the current study have been reported previously [10]. In summary, in the LDS, $\mathrm{NaCl}$ supplementation reduced the anti-albuminuric effect of telmisartan with or without HCT from $42.3 \%$ (placebo) to $9.5 \%(p=0.004)$. However, in $\mathrm{HDS}, \mathrm{NaCl}$ supplementation did not reduce the AER response to telmisartan with or without $\mathrm{HCT}$ (placebo $30.9 \%, \mathrm{NaCl} 28.1 \%, p=0.7)$. The combined effects of treatment and $\mathrm{NaCl}$ vs placebo supplementation on changes in MAP and AER are shown in Fig. 4.

Adverse events Despite a high compliance rate as assessed by counting $\mathrm{NaCl}$ or placebo capsules dispensed throughout the study, some patients initially experienced gastrointestinal side effects, mainly nausea and some vomiting, with $\mathrm{NaCl}$ capsules. However, some participants also reported gastrointestinal symptoms while taking placebo capsules. Despite this, only one patient discontinued the study because of gastrointestinal side effects. The frequency distribution of urinary sodium excretion in HDS and LDS groups during placebo or $\mathrm{NaCl}$ supplementation shows an increase in sodium excretion in both HDS and LDS groups during $\mathrm{NaCl}$ supplementation (ESM Fig. 2).

\section{Discussion}

The major finding in this study was that dietary $\mathrm{NaCl}$ supplementation significantly blunted the antihypertensive effects of telmisartan alone and telmisartan combined with HCT, this effect being independent of habitual low or high salt intake. The systolic BP response to telmisartan with or without HCT was reduced by approximately $50 \%$ with $\mathrm{NaCl}$ supplementation. The addition of HCT increased the antihypertensive effect of telmisartan on systolic BP by only approximately $35 \%$, irrespective of $\mathrm{NaCl}$ supplementation, in both the HDS and LDS groups. The addition of HCT did not offset the effects of $\mathrm{NaCl}$ supplementation. 
a

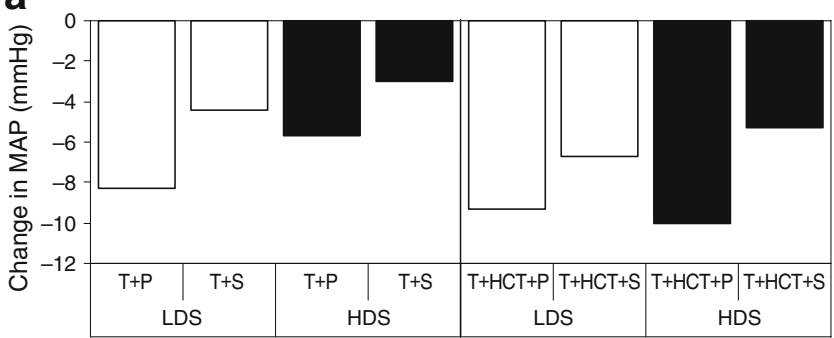

b

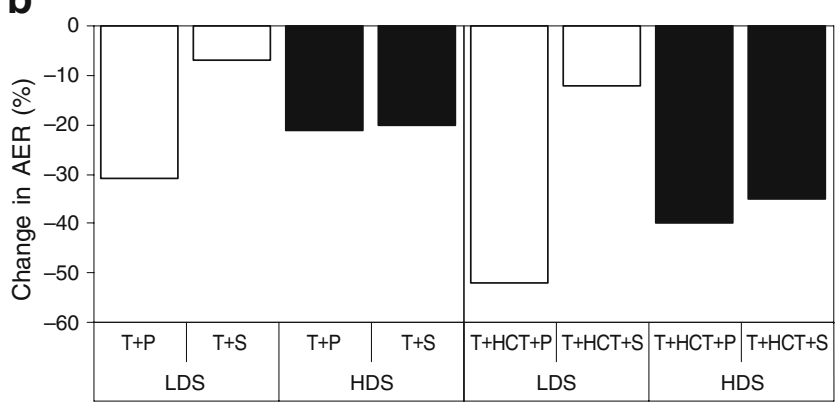

Fig. 4 (a) Changes in MAP (mmHg) and (b) in AER (\% decrease) from baseline according to treatment and habitual salt intake groups. In patients with $\mathrm{HDS}$ or LDS, $\mathrm{NaCl}$ supplementation halved the blood pressure response to telmisartan with or without HCT. In contrast, the AER response to the same therapy was blunted by $\mathrm{NaCl}$ supplementation only in the LDS group. T, telmisartan $40 \mathrm{mg} /$ day; $\mathrm{T}+\mathrm{HCT}$, telmisartan $40 \mathrm{mg}$ /day+hydrochlorothiazide $12.5 \mathrm{mg} /$ day; P, placebo; $\mathrm{S}$, sodium chloride supplementation, $\mathrm{NaCl} 100 \mathrm{mmol} / 24 \mathrm{~h}$. Figure adapted with permission from Ekinci et al. [10]

In contrast to the blood pressure response, the AER response to telmisartan with and without HCT was blunted by $\mathrm{NaCl}$ supplementation only during habitual low salt intake [10]. This implies that renal albumin handling can be modulated by $\mathrm{NaCl}$ intake when the RAS is unsuppressed (LDS) and is unresponsive to $\mathrm{NaCl}$ intake when the RAS is suppressed (HDS). Furthermore, the changes in AER were independent of changes in blood pressure, highlighting potential BP-independent effects of dietary salt intake on AER in this population. The clinical importance of these findings is that short-term excessive salt intake, as simulated with $\mathrm{NaCl}$ supplementation for 2 weeks, had deleterious effects on blood pressure (in HDS and LDS groups) and on AER response (in LDS group) to RAS inhibition and thiazide diuretic therapy in patients with type 2 diabetes.

Early studies in the general population [14-16] and in patients with type 2 diabetes without nephropathy [17] have shown that salt restriction increases the efficacy of several antihypertensive drugs. Similar results have been reported in early and late renal disease. Our group has shown that salt restriction doubles the blood pressure-lowering effects of losartan in patients with type 2 diabetes and increased AER [12]. In the setting of advanced non-diabetic renal disease, a recent study has shown a stepwise decrease in MAP during administration of placebo, losartan or losartan plus HCT, with the greatest reductions in MAP occurring while participants were randomised to a low-salt diet [18]. These data complement the present results and indicate that the relationship between salt intake and antihypertensive therapy represents a continuum ranging from potentiation of the blood pressure response during low salt intake to blunting of the response during high salt intake.

Limitations of the present study include the small number of study participants. In addition, the mean urinary sodium excretion in the LDS group did not meet the National Heart Foundation of Australia guidelines on urinary sodium excretion of $<100 \mathrm{mmol} / 24 \mathrm{~h} \mathrm{[19]}$. However, there was still a large difference in baseline urinary sodium excretion between the HDS and the LDS groups (126 and $256 \mathrm{mmol} / 24 \mathrm{~h}$, respectively). The mean increment in urinary sodium excretion with $\mathrm{NaCl}$ supplementation was $56 \mathrm{mmol} / 24 \mathrm{~h}$, with oral $\mathrm{NaCl}$ supplementation of $100 \mathrm{mmol} / 24 \mathrm{~h}$. In a previous study, supplementation with $80 \mathrm{mmol} / 24 \mathrm{~h}$ of a sustained release preparation of sodium increased urinary sodium excretion by $57 \mathrm{mmol} / 24 \mathrm{~h}$ [17]. The difference between intake and urinary excretion of sodium may be secondary to gastrointestinal side effects of the $\mathrm{NaCl}$ capsules or to net salt retention. However, there was no significant change in body weight during $\mathrm{NaCl}$ supplementation to suggest retention of $\mathrm{NaCl}$.

Blood pressure levels in patients with diabetes have been observed to be more salt-sensitive than in those without diabetes $[20,21]$. This may in part be related to the fact that exchangeable sodium is significantly increased in patients with diabetes $[8,9]$. Furthermore, in vitro studies suggest that hyperglycaemia may lead to activation of serum- and glucocorticoid-regulated kinase-1 signalling, which may lead to increased sodium re-absorption in the kidneys, potentially exacerbating the hypertension seen in diabetes [22]. In the current study, we showed that $\mathrm{NaCl}$ supplementation blunts the antihypertensive effects of telmisartan with or without HCT by approximately $50 \%$. The blunting effect of $\mathrm{NaCl}$ supplementation was homogenous in the HDS and LDS groups, suggesting that results were not influenced by variations in the blood pressure response of individual participants to the same $\mathrm{NaCl}$ load. Although salt sensitivity was not assessed specifically in this study, an analysis of the Dietary Approaches to Stop Hypertension (DASH) study has shown that it is difficult to identify individuals as 'sodium responders' or as 'salt-sensitive', and that recommendations for lower salt intake should be directed at the general public rather than susceptible individuals [23, 24].

In summary, we have shown that the antihypertensive effects of telmisartan with or without HCT are halved with $\mathrm{NaCl}$ supplementation in patients with type 2 diabetes, hypertension and microalbuminuria. By comparison, a meta-analysis of randomised trials has shown that 
in individuals with elevated blood pressure, moderate reduction in dietary salt intake over four or more weeks is an effective way of reducing blood pressure [25]. The mean decrease in systolic BP was $5.1 \mathrm{mmHg}$, with a reduction in urinary sodium of $78 \mathrm{mmol} / 24 \mathrm{~h}$. Similarly, a recent study showed that moderate sodium restriction corresponding to a $55 \mathrm{mmol} / 24 \mathrm{~h}$ decrease in urinary sodium excretion reduced clinic blood pressure by $4.8 \mathrm{mmHg}$ and daytime ABP by $4.7 \mathrm{mmHg}$ in three ethnic groups [26]. The current study in patients with type 2 diabetes and hypertension supports these findings, since an increase in urinary sodium of $56 \mathrm{mmol} / 24 \mathrm{~h}$ during $\mathrm{NaCl}$ supplementation was associated with a $5.6 \mathrm{mmHg}$ blunting of the systolic BP response to telmisartan with or without HCT. Taken together with $\mathrm{NaCl}$ restriction studies, which have shown opposite effects on systolic BP, these results indicate that changes of $\pm 10 \mathrm{mmol} \mathrm{NaCl}$ in daily intake translate into $\pm 1 \mathrm{mmHg}$ changes in systolic BP. This study highlights the importance of dietary salt intake in the management of hypertension in patients with type 2 diabetes. Reducing salt intake on a population basis with collaboration of the food industry would appear to be the most effective way to achieve a reduction in dietary salt intake, as approximately $75 \%$ of dietary salt intake is contained in processed foods $[27,28]$. Organisations such as the World Action on Salt and Health (WASH) programme [29] are working to reduce dietary salt consumption in the general population. This approach is particularly relevant in the context of the two- to fourfold increase in cardiovascular and renal risk in people with type 2 diabetes.

Acknowledgements We thank A. Akdeniz, J. Winikoff, M. Millard and the staff of the Vascular, Laboratory and Clinical Pharmacology Departments at Austin Health for their assistance. In addition, we would like to thank the patients who participated in this demanding study. This work was supported by a Pfizer Cardiovascular-Lipid grant and an unconditional educational grant from Boehringer Ingelheim. During the period of this work, E. I. Ekinci was supported by grants from the Austin Hospital Medical Research Foundation and the National Health and Medical Research Council.

Duality of interest The authors declare that there is no duality of interest associated with this manuscript.

\section{References}

1. Murray CJ, Lauer JA, Hutubessy RC et al (2003) Effectiveness and costs of interventions to lower systolic blood pressure and cholesterol: a global and regional analysis on reduction of cardiovascular-disease risk. Lancet 361:717-725

2. MacIsaac RJ, Barit D, Jerums G (2009) Blood pressure lowering and outcomes in type 2 diabetes: implications of the blood pressurelowering arm of the Advance trial. Curr Hypertens Rev 5:168-180

3. Mancia G, Grassi G (2002) Systolic and diastolic blood pressure control in antihypertensive drug trials. J Hypertens 20:1461-1464
4. Chobanian AV, Bakris GL, Black HR et al (2003) The seventh report of the Joint National Committee on Prevention, Detection, Evaluation, and Treatment of High Blood Pressure: the JNC 7 report. JAMA 289:2560-2572

5. He FJ, MacGregor GA (2003) Salt, blood pressure and the reninangiotensin system. J Ren Ang Ald Syst 4:11-16

6. Karppanen H, Mervaala E (2006) Sodium intake and hypertension. Prog Cardiovasc Dis 49:59-75

7. Adrogue HJ, Madias NE (2007) Sodium and potassium in the pathogenesis of hypertension. N Engl J Med 356:1966-1678

8. O'Hare JA, Ferriss JB, Brady D, Twomey B, O'Sullivan DJ (1985) Exchangeable sodium and renin in hypertensive diabetic patients with and without nephropathy. Hypertension 7:II43-II48

9. Beretta-Piccoli C, Weidmann P (1982) Body sodium-blood volume state in nonazotemic diabetes mellitus. Miner Electrolyte Metab 7:36-47

10. Ekinci EI, Thomas G, Thomas D et al (2009) Effects of salt supplementation on the albuminuric response to telmisartan with or without hydrochlorothiazide therapy in hypertensive patients with type 2 diabetes are modulated by habitual dietary salt intake. Diabetes Care 32:1398-1403

11. Verhave JC, Hillege HL, Burgerhof JG et al (2004) Sodium intake affects urinary albumin excretion especially in overweight subjects. J Intern Med 256:324-330

12. Houlihan CA, Allen TJ, Baxter AL et al (2002) A low-sodium diet potentiates the effects of losartan in type 2 diabetes. Diabetes Care 25:663-671

13. Bach LA, Sharpe K (1989) Sample size for clinical and biological research. Aust N Z J Med 19:64-68

14. Erwteman TM, Nagelkerke N, Lubsen J, Koster M, Dunning AJ (1984) Beta blockade, diuretics, and salt restriction for the management of mild hypertension: a randomised double blind trial. Br Med J (Clin Res Ed) 289:406-409

15. Singer DR, Markandu ND, Cappuccio FP, Miller MA, Sagnella GA, MacGregor GA (1995) Reduction of salt intake during converting enzyme inhibitor treatment compared with addition of a thiazide. Hypertension 25:1042-1044

16. Singer DR, Markandu ND, Sugden AL, Miller MA, MacGregor GA (1991) Sodium restriction in hypertensive patients treated with a converting enzyme inhibitor and a thiazide. Hypertension 17:798-803

17. Dodson PM, Beevers M, Hallworth R, Webberley MJ, Fletcher RF, Taylor KG (1989) Sodium restriction and blood pressure in hypertensive type II diabetics: randomised blind controlled and crossover studies of moderate sodium restriction and sodium supplementation. BMJ 298:227-230

18. Vogt L, Waanders F, Boomsma F, de Zeeuw D, Navis G (2008) Effects of dietary sodium and hydrochlorothiazide on the antiproteinuric efficacy of losartan. J Am Soc Nephrol 19:9991007

19. National Heart Foundation of Australia (2006) Position Statement: The relationships between dietary electrolytes and cardiovascular disease. Available from www.heartfoundation.org.au/SiteCollection Documents/NHFA\%20Dietary\%20Electrolytes\%20CVD\%20QAGeneral.pdf, Accessed 7 November 2009.

20. Strojek K, Grzeszczak W, Lacka B, Gorska J, Keller CK, Ritz E (1995) Increased prevalence of salt sensitivity of blood pressure in IDDM with and without microalbuminuria. Diabetologia 38:1443-1438

21. Tuck M, Corry D, Trujillo A (1990) Salt-sensitive blood pressure and exaggerated vascular reactivity in the hypertension of diabetes mellitus. Am J Med 88:210-216

22. Hills CE, Squires PE, Bland R (2008) Serum and glucocorticoid regulated kinase and disturbed renal sodium transport in diabetes. J Endocrinol 199:343-349

23. Obarzanek E, Proschan MA, Vollmer WM et al (2003) Individual blood pressure responses to changes in salt intake: results from the DASH-Sodium trial. Hypertension 42:459-467 
24. Appel LJ, Giles TD, Black HR et al (2009) ASH position paper: dietary approaches to lower blood pressure. J Clin Hypertens (Greenwich) 11:358-368

25. He FJ, MacGregor GA. Effect of longer-term modest salt reduction on blood pressure. Cochrane Database Syst Rev 2004, Issue 1. Art. No.: CD004937. doi:10.1002/14651858. CD0 04937

26. He FJ, Marciniak M, Visagie E et al (2009) Effect of modest salt reduction on blood pressure, urinary albumin, and pulse wave velocity in white, black, and Asian mild hypertensives. Hypertension 54:482-488

27. James WP, Ralph A, Sanchez-Castillo CP (1987) The dominance of salt in manufactured food in the sodium intake of affluent societies. Lancet 1:426-429

28. Mattes RD, Donnelly D (1991) Relative contributions of dietary sodium sources. J Am Coll Nutr 10:383-393

29. World Action on Salt and Health. Available from www.world actiononsalt.com/, accessed 7 November 2009. 